\title{
Protocole de coopération infirmier et infirmier de pratiques avancées : quelles pistes pour la médecine d'urgence?
}

\author{
Cooperation Protocol and Advanced Practice Nurse: Which Way Forward for Emergency \\ Medicine?
}

\section{B. Riou}

Reçu le 19 septembre 2019; accepté le 19 septembre 2019

(C) SFMU et Lavoisier SAS 2019

La crise observée récemment dans les structures d'urgences et les mouvements sociaux qui l'accompagnent sont avant tout le signe d'une maladie profonde de notre système de santé avec des établissements publics de santé qui font face depuis des années à un étranglement budgétaire [1], une politique de fermeture de lits pilotée par la contrainte budgétaire et une offre de soins en médecine de premier recours qui s'étiole. Quand la ministre de la Santé qualifie notre système de santé comme étant « à bout de souffle », nos structures d'urgences sont asphyxiées et débordées par tout ce que les autres acteurs du système de santé ne veulent plus ou ne peuvent plus faire [2]. Face à cette situation, la ministre de la Santé, Mme Agnès Buzyn, a proposé de développer les protocoles de coopération infirmiers aux urgences et de créer des infirmiers de pratiques avancées (IPA) en médecine d'urgence [3]. Ces propositions paraissent logiques quand on constate le rôle croissant de métiers intermédiaires de la santé dans les autres pays, entre infirmiers et médecins. Surtout, elles ont le mérite de pouvoir être mises en place à relativement court terme quand il faut plus de dix ans pour former un médecin. Comment les IPA pourraient s'intégrer dans la médecine d'urgence de demain?

\section{Protocoles de coopération infirmiers}

L'ensemble des dispositifs réglementaires qui permettent à un infirmier de réaliser des soins qui ne relèvent pas de son décret de compétence, sous la responsabilité d'un médecin, et dans le cadre d'un protocole de coopération entre professionnels de santé dûment autorisé, existent depuis de nombreuses

B. Riou $(\square)$

Sorbonne Université, Service d'accueil des urgences,

Groupe hospitalier Pitié-Salpêtrière,

Assistance publique-Hôpitaux de Paris (AP-HP),

47-83, boulevard de 1'Hôpital, F-75651 Paris cedex 13, France

e-mail : bruno.riou@aphp.fr années [4]. C'est ainsi que des infirmiers peuvent pratiquer des ponctions de moelle osseuse en hématologie, poser des chambres implantables ou des cathéters veineux centraux en anesthésie-réanimation. Si le développement de ces protocoles a été très lent, c'est que le processus administratif d'autorisation est complexe et que sa lenteur (quatre ans et parfois plus) en a désespéré ou découragé plus d'un. L'absence de valorisation financière de la compétence ainsi obtenue a aussi limité les ardeurs et poussé les syndicats infirmiers à s'y opposer, et on peut les comprendre. Ce dernier point vient d'être corrigé très récemment [5], et il faut remercier l'Assistance Publique-Hôpitaux de Paris et son directeur général Martin Hirsch d'avoir été un des premiers à demander cette valorisation auprès du ministère. Cette valorisation est en effet le vecteur d'une amélioration de la rémunération des infirmiers. Et les urgences sont effectivement un lieu idéal où ces protocoles pourraient se développer : prescription anticipée de radiographie [6], réalisation de sutures simples, de plâtres, retrait de dispositif d'immobilisation en traumatologie, etc. Il reste néanmoins des améliorations qui me semblent essentielles pour permettre un large déploiement de ces protocoles :

- simplifier et accélérer le système d'autorisation en modélisant au niveau national avec les sociétés savantes (comme la Société française de médecine d'urgence, SFMU) et le Conseil de l'ordre des médecins, les protocoles nationaux ayant été récemment introduits dans le Code de la santé publique [7] ;

- faciliter l'accès à la formation, préalable indispensable et nécessaire, pour permettre aux professionnels d'adhérer à ces protocoles.

\section{Infirmiers de pratiques avancées (IPA)}

Les IPA sont de création beaucoup plus récente, tout du moins en France, qui est un pays en retard sur à peu près tous les sujets qui concernent les personnels paramédicaux. 
Il s'agit d'une formation de deux ans, dispensée par les universités, permettant d'obtenir le grade de master 2, accessible aux infirmiers ayant déjà une expérience professionnelle et permettant d'acquérir une véritable émancipation par rapport au décret de compétence des infirmiers, dans le cadre d'une spécialité et non plus d'un acte limité comme pour les protocoles de coopération. Les premiers IPA formés terminent leur cursus cette année dans les spécialités suivantes : pathologies chroniques stabilisées : prévention et polypathologies courantes en soins primaires; maladie rénale chronique, dialyse, transplantation rénale; oncologie et hémato-oncologie [8]. La spécialité psychiatrie et santé mentale vient d'être ajoutée cette année. Les statuts de l'IPA n'ont malheureusement toujours pas été publiés, ce qui empêche un établissement de santé de les engager sur une grille appropriée de la fonction publique. Faire deux ans d'études pour gagner autant serait particulièrement regrettable et assez caractéristique du manque de considération envers les soignants !

Les IPA constituent très certainement une solution au moins partielle à la crise de la démographie médicale que nous connaissons actuellement [9]. Mais c'est aussi une évolution logique face à la complexité de plus en plus grande des soins : nous avons besoin, dans de très nombreuses spécialités médicales, de métiers intermédiaires spécialisés entre infirmiers et médecins.

La SFMU devrait se pencher sur le rôle de ces IPA en médecine d'urgence. L'IPA pourrait participer à la régulation médicale des Samu-Centre 15, en collaboration avec le médecin régulateur pour faciliter les admissions directes dans les services d'aval, coordonner et mettre en place des parcours de soins complexes. Une autre cible évidente serait les transports infirmiers interhospitaliers (TIIH) qui existent déjà, pratiqués par des infirmiers qui ont une activité régulière en Smur. L'utilisation d'IPA de médecine d'urgence dans des centres de proximité dépourvus de médecin et travaillant dans le cadre d'un réseau territorial de médecine d'urgence me semble une piste intéressante. J'ai plus de mal à concevoir son rôle dans une équipe hospitalière des urgences particulièrement lorsqu'on considère que de nombreuses tâches jusqu'ici avancées (prescription d'imagerie, sutures, plâtres, etc.) relèvent essentiellement de protocoles de coopération et non des IPA. Toutefois, la réflexion que devra porter la médecine d'urgence sur le rôle de ces IPA ne doit pas s'autocensurer dans un premier temps, quitte à éliminer ensuite des pistes qui se révéleraient finalement peu efficientes ou non prioritaires.

\section{Perspectives et vigilance}

À présent, il convient de donner quelques limites à ceux qui considèrent que les IPA en médecine d'urgence sont une panacée et, sans naïveté, poser la question des raisons qui ont pu amener le ministère de la Santé à accepter si facilement cette proposition de Samu-Urgences de France, dans une période de mouvement social touchant plus d'un tiers des services d'urgence. La première limite est qu'il s'agit d'une réponse dont les effets ne se dessineront que d'ici deux ou trois ans. Il est trop tard pour l'année universitaire 2019-2020, et les infirmiers ayant déjà un master 1 ou pouvant bénéficier de la validation des acquis, donc susceptibles d'être formés en une année, ne sont pas les plus nombreux. Les facultés de médecine et de santé, déjà surchargées par les réformes du troisième cycle, du premier cycle et maintenant du deuxième cycle des études médicales, menées à marche forcée, sans aucun moyen supplémentaire, vont peut-être avoir du mal à se mobiliser et mobiliser leurs enseignants pour créer cette formation. Le financement de ces années d'études reste aussi problématique. Par ailleurs, rien ne dit qu'il s'agira de personnels supplémentaires, positionnés effectivement là où ils pourront dégager du temps médical, en préhospitalier dans les TIIH ou dans des structures de proximité dépourvues de médecin. Le scénario selon lequel les IPA viendraient remplacer des IDE, à moyens quasi constants, avec la difficulté de gérer des équipes ainsi constituées, est malheureusement loin d'être exclu par ces temps de restriction budgétaire des hôpitaux. Surtout, l'effet sur les structures d'urgences sera peut-être intéressant, mais les IPA qu'il conviendrait de former, pour réellement désengorger les urgences hospitalières, concernent prioritairement d'autres domaines, notamment la médecine de premier recours, la protection maternelle et infantile et la gériatrie. Certaines de ces spécialités médicales ne montrent, pour l'instant, guère de volonté de tenter l'aventure, le ministère de la Santé non plus d'ailleurs. Enfin, les efforts déployés par les ministères vers la création des IPA restent lents et timorés, certainement pas à la hauteur des enjeux démographiques actuels de notre système de santé en crise.

Liens d'intérêts : le Pr Bruno Riou est Doyen de la faculté de médecine de Sorbonne Université qui organise des formations d'infirmiers de pratiques avancées.

\section{Références}

1. Juven PA, Pierru F, Vincent F (2019) La casse du siècle. À propos des réformes de l'hôpital public. Collection Raison d'Agir, 192 p

2. Riou B (2019) Tribune : aux urgences, les couloirs de la honte aux urgences. Libération du 30 juin 2019. https://www.liberation.fr/ debats/2019/06/30/aux-urgences-les-couloirs-de-la-honte_1737152 (Dernier accès le 17 septembre 2019)

3. Buzyn A (2019) Discours d'Agnès Buzyn : pacte de refondation des urgences, 9 septembre 2019. https://solidarites-sante.gouv.fr/ actualites/presse/discours/article/discours-d-agnes-buzyn-pacte-derefondation-des-urgences (Dernier accès le 17 septembre 2019)

4. Haute Autorité de santé (2014) Protocole de coopération entre professionnels de santé. Guide méthodologique. https://www.hassante.fr/jcms/c_1240280/fr/protocole-de-cooperation-entre-professionnels-de-sante (Dernier accès le 17 septembre 2019) 
5. République française (2019) Décret $n^{\circ}$ 2019-934 du 6 septembre 2019 portant attribution d'une prime de coopération à certains professionnels de santé exerçant dans le cadre des protocoles de coopération. https://www.legifrance.gouv.fr/eli/decret/2019/9/6/ SSAH1920634D/jo/texte (Dernier accès le 17 septembre 2019)

6. Valentian M, Mewasing BI, Burggraff E, et al (2017) Intérêt d'une demande anticipée de radiographies par l'infirmière organisatrice de l'accueil dans les traumatismes de cheville. Ann Fr Med Urgence 7:77-84

7. République française (2018) Code la santé publique : article L4011. Protocoles nationaux https://www.legifrance.gouv.fr/affichCode.do;jsessionid=2BB2714DCE0A03520248C933D2C0BDC2.
tplgfr41s_2?idSectionTA=LEGISCTA000038842589\&cidTexte $=$ LEGITEXT000006072665\&dateTexte $=20190918$ (Dernier accès le 17 septembre 2019)

8. République française (2018) Décret $\mathrm{n}^{\mathrm{o}}$ 2018-629 du 18 juillet 2018 relatif à l'exercice infirmier en pratique avancée https:// www.legifrance.gouv.fr/affichTexte.do?cidTexte $=$ JORFTEXT 000037218115\&categorieLien=id (Dernier accès le 17 septembre 2019)

9. Riou B, Uzan S (2019) Opinion : des super-infirmiers contre les déserts médicaux. Le Journal du dimanche 28 février 2018. https:// www.lejdd.fr/Societe/Sante/des-super-infirmiers-contre-lesdesertsmedicaux-3585062 (Dernier accès le 17 septembre 2019) 